\title{
THE SPECTRAL TYPE OF SUMS OF OPERATORS ON NON-HILBERTIAN BANACH LATTICES
}

\author{
IAN DOUST ${ }^{凶}$ and GILLES LANCIEN \\ (Received 19 August 2005; accepted 15 September 2006) \\ Communicated by A. J. Pryde
}

\begin{abstract}
T. A. Gillespie showed that on a Hilbert space the sum of a well-bounded operator and a commuting real scalar-type spectral operator is well-bounded. A longstanding question asked whether this might still hold true for operators on $L^{p}$ spaces for $1<p<\infty$. We show here that this conjecture is false. Indeed for a large class of reflexive spaces, the above property characterizes Hilbert space.
\end{abstract}

2000 Mathematics subject classification: primary 47B40.

Keywords and phrases: well-bounded operators, scalar-type spectral operators, functional calculus.

\section{Introduction}

There is a significant body of results which relate the spectral decomposition properties, or the functional calculus properties, of the sum of two commuting operators to those of the summands. Two much-studied classes are the scalar-type spectral operators and the well-bounded operators. For simplicity, let $X$ be a reflexive Banach space. On such a space, an operator $T$ is scalar-type spectral if and only if it admits an integral representation with respect to a countably additive projectionvalued measure or, equivalently, if it admits a $C(\sigma(T))$ functional calculus. An operator $T \in B(X)$ is well-bounded if it admits a Riemann-Stieltjes-type integral representation with respect to an increasing family of projections on $X$ or, equivalently, if it admits an $A C[a, b]$ functional calculus. (Fuller definitions are given in Section 2.) The distinction between these classes is that the spectral expansions for a scalartype spectral operator are of an unconditional nature, while those for well-bounded operators correspond to conditional decompositions of the Banach space.

McCarthy $[13,14]$ proved that the sum and product of two commuting scalar-type spectral operators acting on an $L^{p}(1 \leq p \leq \infty)$ space is again scalar-type spectral. This result has been extended to a wide class of other Banach spaces, including, for

(c) 2008 Australian Mathematical Society 1446-7887/08 \$A2.00+0.00 
example, weakly complete Banach lattices (see [6]). On the other hand, there are reflexive spaces (indeed even UMD spaces), such as the von Neumann-Schatten $C_{p}$ spaces, on which the sum of two commuting scalar-type spectral operators may fail to be scalar-type spectral. More recently, it was shown in [2] that on spaces with Kalton and Weis' property $(\Delta)$ the sum of two commuting scalar-type spectral operators is, however, always well-bounded. The class of spaces with property $(\Delta)$ includes not only all UMD spaces but also those with analytic UMD [8]. As was noted in [2], this result can not be extended to all reflexive spaces.

Gillespie [5] showed that even on $\ell^{2}$, the sum of two commuting well-bounded operators need not be well-bounded. In a positive direction however, he showed that on this space, the sum and product of a real scalar-type spectral operator (that is, a scalar-type spectral operator with real spectrum) and a commuting well-bounded operator are always well-bounded. It had been asked whether this last result might hold on a wider class of spaces such as the reflexive $L^{p}$ spaces. (This question appears explicitly in [2, Section 2], although its consideration significantly predates this reference.) In [2] it was shown that a sufficient condition for the sum of a real scalar-type spectral operator $S$ and a commuting well-bounded operator $T$ to again be well-bounded is that the spectral family of projections associated with $T$ be $R$ bounded.

The aim of this paper is to adapt part of the construction given by Kalton and the second author in [7] to produce counterexamples to the above question on any nonHilbertian reflexive Banach lattice.

\section{Well-bounded operators}

An operator $T \in B(X)$ is well-bounded if it admits an $A C(J)$ functional calculus for some compact interval $J \subset \mathbb{R}$. Here $A C(J)$ denotes the space of absolutely continuous functions on $J=[a, b]$, which is a Banach algebra equipped with the norm $\|f\|_{A C(J)}=|f(b)|+\operatorname{var}_{J} f$. Thus, $T$ is well-bounded if there exists some constant $K$ and a compact interval $J$ such that $\|f(T)\| \leq K\|f\|_{A C(J)}$. We use the notation

$$
\|T\|_{W B}:=\sup \left\{\|f(T)\|: f \in A C[a, b] \text { and }\|f\|_{A C(J)}=1\right\} .
$$

Standard results about well-bounded operators ensure that this quantity depends only on $T$ and not on the choice of interval.

Associated to each well-bounded operator on a reflexive Banach space is a uniformly bounded family of projections known as a spectral family. If a well-bounded operator $T$ has associated spectral family $\{E(\lambda)\}_{\lambda \in \mathbb{R}}$, then for all $f \in A C(J)$,

$$
f(T)=\int_{J}^{\oplus} f(\lambda) d E(\lambda) .
$$

The integral here is a Riemann-Stieltjes-type integral.

We refer the reader to [4] for further details (and the appropriate definitions for nonreflexive spaces) of the theory of well-bounded and scalar-type spectral operators. 
Background definitions concerning bases in Banach spaces and Banach lattices may be found in [10] and [11].

\section{The main result}

The first and main step is to consider the case of a reflexive Banach space with an unconditional basis.

THEOREM 3.1. Let $X$ be a reflexive Banach space which is not isomorphic to $\ell^{2}$. If $X$ admits an unconditional basis, then there exist a real scalar-type spectral operator $S \in B(X)$ and a well-bounded operator $T \in B(X)$ such that $S T=T S$ and $S+T$ is not well-bounded.

PROOF. It was shown in [12] (see the remark at the end of that paper) that if a Banach space $X$ has a normalized unconditional basis and every normalized unconditional basis of $X$ is symmetric, then $X$ is isomorphic to one of $c_{0}, \ell^{1}$ or $\ell^{2}$. It follows that if a reflexive space $X$ which is not isomorphic to $\ell^{2}$ has an unconditional basis, then $X$ admits a normalized unconditional basis which is not symmetric. In particular, $X$ must admit a normalized unconditional basis $\left(x_{n}\right)$ which is not equivalent to any of the standard bases of $c_{0}$ or $\ell^{p}(1 \leq p<\infty)$.

Then [9, Proof of Theorem 4] (see also [10, Theorem 2.a.10]) shows that there exists a permutation $\pi$ and a block basis $\left(v_{n}\right)$ of $\left(x_{\pi(n)}\right)$ whose closed linear span is not complemented. To simplify the notation we may assume that $\left(v_{n}\right)$ is in fact a block basis of $\left(x_{n}\right)$, with

$$
v_{n} \in \operatorname{span}\left(x_{r_{n}+1}, \ldots, x_{r_{n+1}}\right),
$$

where $\left(r_{n}\right)$ is a suitably chosen increasing sequence of integers. Write $X_{n}$ for $\operatorname{span}\left(x_{r_{n}+1}, \ldots, x_{r_{n+1}}\right)$, and let $P_{n}$ denote the projection from $X$ onto $X_{n}$ determined by the basis projections. Let $\alpha_{n}=n^{-2}$. The unconditionality of $\left(x_{n}\right)$ implies that $S:=\sum_{n=1}^{\infty} \alpha_{n} P_{n}$ is a real scalar-type spectral operator on $X$.

For each $n$, there is a norm one projection $\widetilde{V}_{n}$ from $X_{n}$ onto the span of $v_{n}$. Let $V_{n}=\widetilde{V}_{n} P_{n}$ and $W_{n}=P_{n}-V_{n}$. Note that $\left(V_{1}, W_{1}, \ldots, V_{n}, W_{n}, \ldots\right)$ defines a Schauder decomposition of $X$.

Let $Q_{1}=V_{1}$ and for $n \geq 2$, let $Q_{n}=W_{n-1}+V_{n}$. Then $\left\{Q_{n}\right\}$ is a sequence of disjoint finite-rank projections. Note that, for all $N$,

$$
\sum_{n=1}^{N} Q_{n}=V_{N}+\sum_{n=1}^{N-1} P_{n},
$$

and so these partial sums are uniformly bounded. Let $\beta_{n}=-n^{-2}-n^{-3}$. It follows from [1, Theorem 3.3] that $T:=\sum_{n=1}^{\infty} \beta_{n} Q_{n}$ converges to a well-bounded operator on $X$.

It is straightforward to verify that for all $m$ and $n, P_{m}$ and $Q_{n}$ commute. It follows that $S$ and $T$ commute. It remains then to show that $S+T$ is not well-bounded. Suppose, to the contrary, that $S+T$ is well-bounded. 
A small calculation shows that

$$
S+T=\sum_{n=1}^{\infty}-\frac{1}{n^{3}} V_{n}+\sum_{n=1}^{\infty} \gamma_{n} W_{n},
$$

where $\gamma_{n}=\alpha_{n}+\beta_{n+1}$ is positive for all $n \geq 1$. Choose $f_{n} \in A C[-1,1]$, for $n \geq 1$, so that $f_{n} \equiv 1$ on $\left[-1,-n^{-3}\right], f_{n} \equiv 0$ on $\left[-(n+1)^{-3}, 1\right]$ and $f_{n}$ decreases linearly on $\left[-n^{-3},-(n+1)^{-3}\right]$. Then $f_{n}(S+T)=\sum_{j=1}^{n} V_{j}$. We now claim that the increasing sequence of projections $\left(\sum_{j=1}^{n} V_{j}\right)_{n=1}^{\infty}$ is unbounded. Otherwise, since $X$ is reflexive, it would converge in the strong operator topology and its limit would be a bounded projection onto the closed linear span of $\left\{v_{n}, n \geq 1\right\}$. Thus, $\left\{\left\|f_{n}(S+T)\right\|\right\}$ is unbounded and, as $\left\|f_{n}\right\|_{A C(J)}=1$ for each $n$, it follows that $S+T$ must not be a well-bounded operator.

The hypotheses in this theorem are not optimal, but they do suffice to cover the cases of most interest. In particular, we have as a first corollary.

Corollary 3.2. Let $1<p<\infty, p \neq 2$, and let $X=L^{p}[0,1]$. Then there exist a real scalar-type spectral operator $S \in B(X)$ and a well-bounded operator $T \in B(X)$ such that $S T=T S$ and $S+T$ is not well-bounded.

PROOF. The Haar system forms an unconditional basis for $X$.

\section{Reflexive Banach lattices}

Finally, we prove the result announced in the introduction.

COROllary 4.1. Let $X$ be a reflexive Banach lattice which is not isomorphic to a Hilbert space. Then there exist a real scalar-type spectral operator $S \in B(X)$ and a well-bounded operator $T \in B(X)$ such that $S T=T S$ and $S+T$ is not well-bounded.

PROOF. We essentially follow [7, Proofs of Theorems 3.5 and 3.7]. Let us assume that for any real scalar-type spectral operator $S \in B(X)$ and any well-bounded operator $T \in B(X)$ commuting with $S$, the sum $S+T$ is well-bounded. We then show that $X$ is order isomorphic to a Hilbert space. For this, since $X$ is reflexive and therefore order continuous, it is enough to prove that every normalized sequence of disjoint elements of $X$ is equivalent to the canonical basis of $\ell^{2}$ (see [11, Lemma 1.b.13]).

So, let $\left(v_{n}\right)$ be a normalized sequence of disjoint elements of $X$. As in [7], we can pick an unconditional Schauder decomposition $\left(X_{n}\right)$ of $X$ such that for each $n, X_{n}$ is an ideal of $X$ and $v_{n} \in X_{n}$. Then, by repeating the argument in the proof of Theorem 3.1, we obtain that $Y=\overline{\operatorname{span}}\left\{v_{n}, n \geq 1\right\}$ is complemented in $X$. Thus, for any real scalartype spectral operator $S \in B(Y)$ and any well-bounded operator $T \in B(Y)$ commuting with $S, S+T$ is well-bounded (indeed, any counterexample on $Y$ could easily be extended into a counterexample on $X)$. Finally, it follows from Theorem 3.1 that $\left(v_{n}\right)$ is equivalent to the canonical basis of $\ell^{2}$. 


\section{REMARKS.}

(1) Virtually identical constructions provide examples where the product rather than the sum fails to be well-bounded; with $\left\{\alpha_{n}\right\},\left\{\beta_{n}\right\},\left\{P_{n}\right\}$ and $\left\{Q_{n}\right\}$ as in the proof of Theorem 3.1, then setting

$$
S=I+\sum_{n=1}^{\infty}\left(e^{\alpha_{n}}-1\right) P_{n} \quad \text { and } \quad T=I+\sum_{n=1}^{\infty}\left(e^{\beta_{n}}-1\right) Q_{n},
$$

will suffice.

(2) It should be noted that on nonreflexive spaces, these questions are somewhat more complicated since the relationship between the functional calculus conditions and the integral representation conditions is not as strong. On spaces such as $\ell^{\infty}$, or indeed any Grothendieck space with the Dunford-Pettis property, the countable additivity requirement for a spectral measure allows very few scalar-type spectral operators. On such a space every scalar-type spectral operator is a finite linear combination of disjoint projections [15] and consequently the sum of a real scalar-type spectral operator and a commuting well-bounded operator is always well-bounded.

(3) More recently, the present authors and F. Lancien [3] have used ideas from [8] to show that if either $X$ or $X^{*}$ is a Grothendieck space, then the sum of a real scalar-type spectral operator and a commuting well-bounded operator is always well-bounded. This settles the question on, in particular, any $L^{1}$ or $C(K)$ space.

(4) On some nonreflexive spaces it is relatively easy to construct explicit examples where the sum of a real scalar-type spectral operator and a commuting wellbounded operator is not well-bounded. For example, on the space of trace class operators $C_{1}$, the operators $S(A)=D_{1} A$ and $T(A)=A D_{2}$, where $D_{1}$ and $D_{2}$ are diagonal matrices with real entries, are commuting scalar-type spectral operators whose sum, in general, will fail to be well-bounded.

\section{References}

[1] Q. Cheng and I. Doust, 'Compact well-bounded operators', Glasg. Math. J. 43 (2001), 457-467.

[2] I. Doust and T. A. Gillespie, 'Well-boundedness of sums and products of operators', J. London Math. Soc. (2) 68 (2003), 183-192.

[3] I. Doust, F. Lancien and G. Lancien, 'Spectral theory for linear operators on $L^{1}$ or $C(K)$ spaces', in: Asymptotic Geometric Analysis, Harmonic Analysis, and Related Topics (Murramarang, 2006), Proceedings of the Centre for Mathematics and its Applications, Australian National University, 42 (Australian National University, Canberra, 2007), pp. 1-10.

[4] H. R. Dowson, Spectral Theory of Linear Operators, London Mathematical Society Monographs, 12 (Academic Press, London, 1978).

[5] T. A. Gillespie, 'Commuting well-bounded operators on Hilbert spaces', Proc. Edinb. Math. Soc. (2) 20 (1976), 167-172.

[6] - 'Boundedness criteria for Boolean algebras of projections', J. Funct. Anal. 148 (1997), 70-85.

[7] N. J. Kalton and G. Lancien, 'A solution to the problem of $L^{p}$-maximal regularity', Math. Z. 235 (2000), 559-568. 
[8] N. J. Kalton and L. Weis, 'The $H^{\infty}$-calculus and sums of closed operators', Math. Ann. 321 (2001), 319-345.

[9] J. Lindenstrauss and L. Tzafriri, 'On the complemented subspaces problem', Israel J. Math. 9 (1971), 263-269.

[10] _ Classical Banach Spaces I (Springer, Berlin, 1977).

[11]_Classical Banach Spaces II (Springer, Berlin, 1979).

[12] J. Lindenstrauss and M. Zippin, 'Banach spaces with unique unconditional basis', J. Funct. Anal. 3 (1969), 115-125.

[13] C. A. McCarthy, 'Commuting Boolean algebras of projections', Pacific J. Math. 11 (1961), 295307.

[14] , 'Commuting Boolean algebras of projections. II. Boundedness in $L_{p}$ ', Proc. Amer. Math. Soc. 15 (1964), 781-787.

[15] W. Ricker, 'Spectral operators of scalar type in Grothendieck spaces with the Dunford-Pettis property', Bull. London Math. Soc. 17 (1985), 268-270.

IAN DOUST, School of Mathematics, University of New South Wales, Sydney,

NSW 2052, Australia

e-mail: i.doust@unsw.edu.au

GILLES LANCIEN, Equipe de Mathématiques UMR 6623, Université de Franche-Comté, F-25030 Besançon cedex, France

e-mail: gilles.lancien@math.univ-fcomte.fr 